This work is licensed under a Creative Commons Attribution 4.0 International License.

Ovaj rad dostupan je za upotrebu pod licencom Creative Commons Imenovanje 4.0 međunarodna.

UDK 821.112.2.09 Herrndorf, W.-31

Zarah RIETSCHEL

Westfälische-Wilhelms-Universität Münster, Deutschland

Schlosspl. 2, 48149 Münster

zarahrietschel@gmail.com
Origineller wissenschaftlicher Beitrag

Original Research Article

Erhalten am 31. 5. 2021

Received: 31 May 2021

Angenommen am 19. 8. 2021

Accepted: 19 August 2021

\title{
DEN TOD MIT DEM TOD BEKÄMPFEN. SUIZID ALS AUSWEG IN WOLFGANG HERRNDORFS ARBEIT UND STRUKTUR
}

\section{Zusammenfassung}

Das vorliegende Paper widmet sich der textnahen Analyse von Wolfgang Herrndorfs autobiographischem Text Arbeit und Struktur hinsichtlich des Zusammenhangs von der Suizidentscheidung des autobiographischen Ich und der ihm gestellten Diagnose eines tödlichen Hirntumors (Glioblastoms). Es lässt sich erkennen, dass die wiederholten Untersuchungen und deren Ergebnisse sowie eigens angestellte Recherchen und das Heranziehen von Statistiken hinsichtlich der Selbsttötungsabsicht als sogenannte ,Entscheidensressourcen' fungieren. Sie nehmen demnach Einfluss auf die dem autobiographischen Ich verbleibende Zeit und zeitgleich auf die Frage nach einem geeigneten Zeitpunkt für den visierten Suizid. Neben der Untersuchung unterschiedlicher Reaktionen auf den fortschreitenden Krankheitsverlauf anhand der Methode des Close Readings nimmt das Paper außerdem eine Parallelisierung der geäußerten Suizidabsicht und des intensiven Arbeitens des autobiographischen Ich als Formen der Bewältigungsstrategie für die diagnostizierte Erkrankung vor. Dem Arbeiten in Form des Schreibens kommt dabei in erster Linie die Rolle einer sinnstiftenden Beschäftigung zu, während der geplante Suizid als ,Exitstrategie fungiert, die als letztmögliche Lösung herangezogen wird, um nicht der Krankheit 
zu erliegen. Während eine Vielzahl von interdisziplinären Diskursen dem Thema Suizid seit jeher mit Ablehnung begegnet, erfährt der Suizid als Krankheit eine historisch verankerte Legitimation, die sich auch in dem untersuchten Text anhand der Reaktionen des sozialen Umfeldes des autobiographischen Ich erkennen lässt. Der unabdingbare Tod angesichts der medizinischen Diagnosen legitimiert somit die Selbsttötung.

Schlüsselwörter: Wolfgang Herrndorf, Arbeit und Struktur, Autobiographie, Suizid, Krankheit

\section{Einleitung}

Am 18.02.2010 erhält der Autor Wolfgang Herrndorf die Diagnose „Hirntumor" (Herrndorf 99), die eine Woche später als Glioblastom spezifiziert wird (ebd. 106). Angesichts eines unvermeidbaren, krankheitsbedingten Todes legt sich Herrndorf bereits circa zweieinhalb Monate nach Erstdiagnose darauf fest, sich der Krankheit durch Suizid zu entziehen, bevor sie ihn seiner Handlungsmacht berauben kann: „Was ich brauche, ist eine Exitstrategie“ (ebd. 50).

Das Entscheiden für einen Tod durch Suizid ist dabei sowohl durch die medizinische Diagnose als auch durch die Angst vor einem krankheitsbedingten Kontrollverlust (ebd. 142), insbesondere in Form einer eingeschränkten Möglichkeit der Kommunikation (ebd. 224) bedingt. Hierdurch etabliert sich ,Zeit als den Suizid beeinflussender Faktor. Entsprechend heißt es in einem späteren Eintrag des Tagebuchblogs Arbeit und Struktur': „Ich weiß, wie, ich weiß, wo, nur das Wann ist unklar. Aber dass ich zwei der Kategorien kontrolliere und die Natur nur eine - ein letzter Triumph des Geistes über das Gemüse“ (ebd. 198). ${ }^{2}$

\footnotetext{
${ }^{1}$ Ursprünglich handelt es sich bei Arbeit und Struktur um einen Tagebuchblog, der online erschien und auch heute noch öffentlich zugänglich ist (vgl. www.wolfgang-herrndorf.de/). Als Grundlage für dieses Paper fungiert allerdings die gedruckte Taschenbuchausgabe des Textes, die nach Herrndorfs Tod bei Rowohlt erschien (vgl. Herrndorf, Wolfgang. Arbeit und Struktur. Rowohlt, 2013).

${ }^{2}$ Den Begriff, Gemüse` nutzt das autobiographische Ich als Synonym für den beschriebenen Zustand fehlender Kontrolle und Kommunikationsmöglichkeit, welcher als erster Motivationsfaktor für das selbstbestimmte Sterben fungiert: „Dieser Scherbenhaufen im Innern bei gleichzeitiger Unfähigkeit zu sprechen, das ist nicht meine Welt. Auch wenn man da möglicherweise noch zwei Gemüsestufen über dem Apalliker rangiert, das geht nicht. Menschliches Leben endet, wo die Kommunikation endet, und das darf nie passieren. Das darf nie ein Zustand sein. Das ist meine größte Angst" (ebd. 224).
} 
Die erste durch eine Freundin recherchierte Lebenserwartung nach Glioblastom-Diagnose liegt bei 17,1 Monaten bzw. 698 Tagen (ebd. 107) und wird zum Dreh- und Angelpunkt für das weitere Vorgehen des autobiographischen Ich. Es beabsichtigt, die verbleibende Zeit mit dem Arbeiten an zu früheren Zeitpunkten begonnenen Romanprojekten zu verbringen (ebd.), nachdem es dieses Urteil durch die Lektüre einer bereits erschienenen Textsammlung überprüft und gefestigt hat: „,Diesseits des Van-Allen-Gürtels' noch mal Korrektur gelesen. Um zu gucken: Lohnt sich das überhaupt? Kann ich das? Oder mache ich lieber eine Weltreise?“ (ebd. 23). So heißt es konkret im Text: „Ich werde noch ein Buch schreiben, egal wie lange ich noch habe. Wenn ich einen Monat habe, schreibe ich eben jeden Tag ein Kapitel. Wenn ich drei Monate habe, wird es ordentlich durchgearbeitet. Ein Jahr ist purer Luxus." (ebd. 107) Hier deutet sich bereits die Funktion an, die dem schriftstellerischen Arbeiten zugeschrieben werden kann: Das Arbeiten fungiert als Beschäftigungs- sowie Bewältigungsstrategie angesichts der diagnostizierten Krankheit. Dieser Umstand erklärt auch den Titel des Textes, der sich aus dem Gespräch des autobiographischen Ich mit einem ebenfalls an einem Glioblastom erkrankten Mann ergibt, der dem autobiographischen Ich zu „Arbeit und Struktur“ rät (ebd. 114). Das Arbeiten verleiht dem Rest des Lebens des autobiographischen Ich Struktur und gibt ihm die Möglichkeit, mit der Diagnose zu leben.

Gleiches gilt für die Entscheidung, sich durch Suizid das Leben zu nehmen, durch die das autobiographische Ich sich seine Handlungsmacht und Selbstbestimmung erhält. Somit teilen sich das schriftstellerische Arbeiten und das Entscheiden für ein selbstbestimmtes Sterben die Funktion der Bewältigungsstrategie, während der Vollzug des Suizids als letzte Option und Exitstrategie fungiert.

Das Zusammenspiel von medizinischen Diagnosen sowie herangezogenen Statistiken, der Arbeit als Bewältigungs- und des Suizids als Exitstrategie stellt dabei den Untersuchungsgegenstand des vorliegenden Papers dar. Ziel des Papers ist es, die Frage zu beantworten, inwiefern sich die medizinischen Diagnosen und der Krankheitsverlauf in diesem Rahmen als Einflussfaktoren auf das Entscheiden für den Suizid lesen lassen. Dabei erfolgt eine literaturwissenschaftliche Analyse des autobiographischen Textes anhand der Methode des Close Reading. Das Medium des Tagebuchblogs ermöglicht diesbezüglich einen zeitdeckenden Einblick in die Gedanken des autobiographischen Ich und dessen Umgang mit der diagnostizierten Krankheit. Vor dem Hintergrund, dass 
die Selbsttötung des Autors Herrndorf das Ende des autobiographischen Textes bedeutet, beschäftigt sich das Paper außerdem mit der Besonderheit des autobiographischen Textes in diesem Zusammenhang, geht weiterreichend auf die moralische Legitimität der Selbsttötung ein und diskutiert vor diesem Hintergrund die historische Sonderstellung des Krankensuizids.

\section{Mit dem Suizid endet der Text}

Zunächst als Informationsblog für seine Freund*innen gedacht, um nicht allen ständig die gleichen Fragen beantworten zu müssen, lässt sich Wolfgang Herrndorf von seinem sozialen Umfeld überzeugen, dass der von ihm eingerichtete Tagebuchblog mit dem Titel Arbeit und Struktur auch der Öffentlichkeit zur Verfügung gestellt werden solle (Gärtner und Passig 443).

Die regelmäßigen Tagebucheinträge beginnen am 08.03.2010 (Herrndorf 9) und reichen bis zum 20.8.2013 (ebd. 425). Sie umfassen somit einen Zeitraum von kurz nach der ersten Diagnose bis sechs Tage vor dem Suizid des Autors und begleiten das autobiographische Ich durch die verschiedenen Stadien seiner Krankheit, die wiederholten Prognosen hinsichtlich seiner krankheitsbedingten Lebenserwartung und die medizinischen Untersuchungsergebnisse sowie den Umgang mit dem bevorstehenden Tod. Bis heute steht der Blog online unter https://www.wolfgang-herrndorf.de/ zur Verfügung. Auf der ersten Seite findet sich die Anmerkung, dass er ursprünglich nicht zur Veröffentlichung gedacht war und hierfür Passagen gekürzt und Namen geändert wurden (Gärtner und Passig 443).

Die Besonderheit des autobiographischen Textes als Medium, in welchem das Thema Suizid behandelt wird, liegt darin, dass der Text mit dem Suizid endet. Die Entscheidung für den Suizid setzt somit nicht nur einen Endpunkt für das Leben des autobiographischen Ich, sondern zugleich das Ende für den Text fest.

Trotz des Verständnisses, dass im autobiographischen Text eine Übereinstimmung von Autor*in, Erzähler*in und Figur besteht (Wagner-Egelhaaf, Autobiografie 6), erfordert die Lektüre eines autobiographischen Textes eine Unterscheidung zwischen autobiographischem Ich und Autor*in. So lässt sich aus der autodiegetischen Erzählsituation eine „subjektive Wahrnehmungsperspektive“ (ebd. 2) ableiten, die "Selbst- wie die Fremdwahrnehmung“ (ebd.) des autobiographischen Ich 
bestimmen kann. Angesichts der Tatsache, dass Autobiographien stets „vom Tod hergeschrieben“ (Wagner-Egelhaaf, „Trauerspiel“ 73) sind, lassen sich Aussparungen sowie die Setzung eines bestimmten Fokus innerhalb eines autobiographischen Textes sowohl auf diese perspektivische Subjektivität als auch auf zeitlich bedingte Veränderungen oder auch Lücken innerhalb der Erinnerungen der Autor*innen zurückführen. Das Tagebuch bildet in dieser Hinsicht eine Ausnahme, da es hier in der Regel keine weiten Abstände zwischen Erleben einer Sache und Verschriftlichung derselben gibt und es entsprechend nicht „retrospektiv“ (Wagner-Egelhaaf, Autobiografie 6) ist. Im Falle von Herrndorfs Tagebuchblog lässt sich eine Besonderheit feststellen, die in der Integration von elf Rückblenden innerhalb des ansonsten kontinuierlich fortschreitenden Textes besteht. ${ }^{3}$

Sie entsprechen somit den Strukturmerkmalen eines autobiographischen Textes, während die Einträge ab dem 08. März 2010 als Tagebucheinträge verfasst und sich somit mit der Zeit decken, in der geschrieben wird. Bei den Rückblenden ist der Prozess des Sich-erinnerns, welcher nicht nur „Rekonstruktion“ von Geschehenem, sondern auch „Konstruktion“ (ebd. 13) von Fiktivem meinen kann, dabei gefragter: „Der mediale Prozess der Erinnerung [...] wird zum Konstitutionselement der autobiographischen Fiktionalität“ (ebd.).

\section{Suizid im Spiegel der Zeit}

Seit jeher wird das Thema ,Selbsttötung' interdisziplinär diskutiert und erhält Einzug in - unter anderem - philosophische, theologische, rechtswissenschaftliche und literaturwissenschaftliche Arbeiten. In literarischen Texten findet es sich dabei bereits im 8. Jahrhundert vor Christus und setzt sich bis heute fort (Vorjans 21), woraus sich ableiten lässt, dass Suizid als Thema die Menschen zu jeder Zeit emotional bewegt und beschäftigt. Insbesondere die Frage der Moralität von Suizid findet Einzug in die unterschiedlichsten Forschungsfelder. Dabei hängt die Legitimation des Suizids von der Beantwortung der Frage ab, wem das Leben der einzelnen Person gehöre, und wer folglich

\footnotetext{
${ }^{3}$ Neun der zehn Rückblenden sind jeweils mit der Überschrift Rückblende versehen und setzen nach den ersten acht Kapiteln zuzüglich des allerersten Kapitels des Textes ein, welches mit dem Titel Dämmerung überschrieben ist (Herrndorf 8). Bei diesem ersten Kapitel handelt es sich dabei ebenfalls um eine Analepse, wobei diese als Kindheitserinnerung deutlich weiter zurückliegt als die anderen beschriebenen Ereignisse. Die mit Rückblende überschriebenen Kapitel beinhalten stattdessen Situationen, die vor Beginn der in den nummerierten Kapiteln erzählten Geschichte stattgefunden haben. Während der erste Tagebucheintrag vom 8.3.2010 stammt, umfassen die Rückblenden eine Zeitspanne vom 25.2.-8.3.2010.
} 
über dessen Ende verfügen dürfe. Die Antwort auf diese Frage variiert im Laufe der Zeit und reicht von göttlichen Instanzen über den Staat und die Gesellschaft bis hin zur Familie.

So spricht sich beispielsweise Platon gegen die Selbsttötung mit der Begründung aus, dass das Leben nicht dem Individuum, sondern den Göttern gehöre (Platon 737), während Aristoteles als Schüler Platons den Eigentümer des Lebens im Staat erkennt (Aristoteles 233). In weiten Teilen der Antike wird die individuelle Suizid-Entscheidung demnach als inakzeptabel angesehen, da der Mensch zu jeder Zeit von externen Instanzen wie Göttern oder den Mitmenschen in einer staatlichen Gemeinschaft abhängig sei (Graefe 25).

Dieser Auffassung stehen andere philosophische Strömungen der Antike wie der Epikureismus und der Stoizismus gegenüber, die sich in erster Linie auf eine „moralisch gute und richtige Lebensführung“ (ebd. 26) konzentrieren, welche die Beendigung des eigenen Lebens unter den Umständen akzeptiert, dass eine entsprechende Lebensführung nicht möglich sei (ebd). So spricht sich beispielsweise Seneca als Vertreter des Stoizismus bereits in der Antike dafür aus, dass die Entscheidung über die Beendigung des eigenen Lebens beim Individuum selbst liege (Giebel 248) und greift hierdurch bereits das sich mit der Aufklärung verbreitende Verständnis des Suizids als Freiheitsakt auf.

Im Mittelalter erfährt der Suizid eine noch stärkere Verurteilung und Ablehnung, die sich auch im Umgang mit Suizidant*innen und ihren Familien in Form von Leichenschändungen, Konfiskationen von Besitztümern und außerkirchlichen Bestattungen zeigt (Minois 26). Obwohl sich auch im Zeitalter der Aufklärung Positionen ausmachen lassen, die den Suizid als Handlung wider die Vernunft ablehnen (Kant 68), etabliert sich das Individuum zunehmend als Entscheidungs-Instanz über das eigene Leben (Graefe 14) und tritt somit neben die bereits genannten Optionen. ${ }^{4}$

Während eine Vielzahl von interdisziplinären Diskursen dem Thema Suizid seit jeher mit Ablehnung begegnet, erfährt der sogenannte „Krankensuizid“, unter den auch der Fall des autobiographischen Ich in Herrndorfs Arbeit und Struktur fällt, eine historisch verankerte Legitimation (Macho 80).

\footnotetext{
${ }^{4}$ Thomas Macho widmet in seiner Monographie Das Leben nehmen ein ganzes Kapitel dieser Frage. 
So steht der Suizid aus Krankheit beispielsweise in der Vormoderne, einem Zeitalter der hegemonialen Ablehnung des Suizids, als sogenannter „Notsuizid[ ]" (ebd.) in einer Reihe von Suiziden, der auch in dieser Zeit bereits mit Verständnis begegnet wird (ebd. 56). Suizide aus „Ehre, Selbstopfer und Not (Krankheit, Hunger, Schmerz, Alter)“ (ebd.) werden geduldet, obwohl das Leben in dieser Zeit nicht als individuelles Gut, sondern lediglich als „Leihgabe“ (ebd. 55) betrachtet wird und aus diesem Grund nicht individuell über dessen Beendigung entschieden werden soll. Der Suizid des autobiographischen Ich in Herrndorfs Arbeit und Struktur kann angesichts der diagnostizierten Erkrankung folglich als „Notsuizid[ ]“ (ebd. 80) gelesen werden, wodurch eine soziale Legitimation der Selbsttötungsabsicht erwartbar wird.

Dass die Suizidentscheidung keinem Todeswunsch geschuldet ist, sondern lediglich aus der Sorge vor einem Zugrundegehen am Glioblastom entspringt, zeigt sich, wenn es in einem sehr frühen Eintrag im Text, in dem es um mögliche Suizidmethoden geht, heißt: „Ich will mir ja gar nichts antun. Das ist doch nicht der Punkt“ (Herrndorf 75).

In Hinblick auf das Erkennen des Suizids als selbstbestimmten und vernünftigen Akt, lässt sich abschließend das Werk Hand an sich legen. Diskurs über den Freitod des österreichischen Schriftstellers Jean Améry anführen. In seinem Werk plädiert Améry für das Verständnis, dass dem Suizid der Gebrauch des Rechts, „sich selbst [zu] gehören“(99) zugrunde liege und die Nutzung desselben in keinem Fall als Handlung wider die Moral degradiert werden könne (ebd. 102). So beantwortet er final die Frage, wem der Mensch gehöre, mit einem überzeugten „sich selbst“ (105, Hervorhebung im Original) und nimmt somit eine Haltung ein, „die vom Selbstbestimmungsrecht des einzelnen und der Freiheit zu entscheiden, was er mit seinem Leben macht, getragen ist“" (Decher 171).

\section{Der Suizid als Lebensentscheidung}

Unter den Begriff, Lebensentscheidungen' werden Entscheidungen gefasst, denen eine besondere Signifikanz für das Leben der sie betreffenden Personen oder, in literarischen Zusammenhängen, Figuren zukommt. Martina Wagner-Egelhaaf zählt hierunter Entscheidungen hinsichtlich der Berufs- sowie Partner ${ }^{\star}$ innenwahl, „religiöse Entscheidungen, Reiseentscheidungen, bei Schriftstellern und Schriftstellerinnen Werkentscheidungen, aber auch Entscheidungen für die Zeit nach dem Tod des Autobiographen beziehungswei- 
se der Autobiographin [...]“ (ebd. 72). Der Suizid bildet in dieser Reihe eine besondere Art der Lebensentscheidung, da er die Beendigung des Lebens bezweckt und somit nicht im Sinne der anderen genannten Lebensentscheidungen dessen weiteren Verlauf beeinflussen kann. So mag es zunächst fraglich erscheinen, ob sich das Entscheiden für die Beendigung des eigenen Lebens als Lebensentscheidung verstehen lässt. Die Entscheidens-Definition, auf welcher die vorliegende Analyse basiert, lässt sich dabei jedoch auch für das Entscheiden zum Suizid fruchtbar machen. So impliziert das Entscheiden die „Erzeugung von alternativen Handlungsoptionen zu einem bestimmten Gegenstand oder Thema, [...] die explizite Bewertung dieser Alternativen, sowie [...] die kontingente Selektion einer dieser Alternativen“" (Pfister 13). Die Handlungsmöglichkeit hinsichtlich der Suizidentscheidung ist dabei, wie auch bei einigen der anderen aufgezählten Lebensentscheidungen, binär: sterben oder weiterleben, heiraten oder nicht heiraten, eine Familie gründen oder nicht.

Die Signifikanz der Entscheidung zum Suizid sowie die hieraus folgende Beendigung des eigenen Lebens bewirkt, dass die Suizidentscheidung zu einer finalen Lebensentscheidung avanciert. Dabei steht der Entscheidensprozess unter einer Vielzahl von Einflussfaktoren, die sich als Entscheidensressourcen motivieren lassen. Unter Entscheidensressourcen werden „Mittel [verstanden], die Entscheiden sowohl ermöglichen als auch bei seinem Vollzug zum Einsatz gelangen“ (ebd. 25). Darunter kann sowohl Gegenständliches fallen als auch Personen oder Abstrakta wie „Wissen, [...] Wertvorstellungen, Programme, Ideologien, [...] Bedürfnisse, Affekte, Emotionen, [...] Macht, Autorität [...]“ (ebd. 26). Indem sie zur Erzeugung alternativer Handlungsmöglichkeiten beitragen und verschiedene Optionen zum Umgang mit der Kontingenz einer Entscheidenssituation liefern (ebd. 25), tragen Entscheidensressourcen demnach aktiv dazu bei, den Prozess des Entscheidens und dessen Charakteristika herauszubilden. Das Motivieren ebensolcher Entscheidensressourcen auch hinsichtlich des Suizids gibt diesen erneut als Folge eines Entscheidensprozesses $\mathrm{zu}$ erkennen. Im Falle des autobiographischen Ich in Arbeit und Struktur tritt insbesondere die Entscheidensressource der medizinischen Untersuchungsergebnisse und somit der Krankheitsverlauf in den Fokus, die ihrerseits Einfluss auf die verbleibende Zeit nehmen, die ebenfalls als Entscheidensressource motiviert werden kann. So finden sich im Text vermehrt Einträge, in denen einer aktuellen Diagnose die Frage nach der verbleibenden Zeit folgt: 
So, sagt Dr. Vier, setzt sich und liest. Aha, aha. Schrankenstörung, Strukturstörung, Balken, kennen wir ja. Leukenzephalopathie und immer wieder das Wort progredient. 5,3 Zentimeter hinten links, mehr oder weniger stabil. Besser geht's doch kaum, behaupte ich, Dr. Viert widerspricht. Aber erst mal drei Monate? Ja, das wohl. Und ab. (beispielhaft Herrndorf 319)

Die Begrenzung der durch die Krankheit bestimmten, verbleibenden Lebenszeit wird zu einem Kriterium, das zunehmend an Einfluss gewinnt, was sowohl Einschränkung als auch Freiheit bedeuten kann. So erkennt Hermann Lübbe die Besonderheit der Entscheidung gegenüber der Wahl darin, dass

[d]ie Entscheidungssituation [...] eine Situation befristeter Dauer [ist]. Daraus folgt umgekehrt, daß man im Verhältnis zu Möglichkeiten, die von sich aus keinen unmittelbar spürbaren Eindruck ausüben, nur dadurch zu einer Entscheidung kommt, daß man sich selber Termine setzt. (23)

Durch die Kopplung des Suizids an einen Zeitpunkt, zu dem das autobiographische Ich noch über ausreichend Kontrolle über sich selbst verfügt (Herrndorf 142), legt es somit einen bestimmten Termin für den Vollzug der Entscheidung für oder gegen den Suizid fest. Der gesetzte Termin wird laut Lübbe zu einem „,point of no return“" (21), dessen Überschreiten automatisch zur Entscheidung führt (ebd.).

\section{Den Tod mit dem Tod bekämpfen: Bewältigungs- und Exitstrategie}

Neben dem Entscheiden für den Tod durch Suizid hängt auch das schriftstellerische Arbeiten des autobiographischen Ich von seinem Krankheitsverlauf, genauer von der hiervon abhängigen verbleibenden Zeit ab.

Dass das schriftstellerische Arbeiten für das autobiographische Ich in Wolfgang Herrndorfs Arbeit und Struktur etwas darstellt, das ihm die Kraft gibt, mit seinem diagnostizierten Glioblastom umzugehen, deutet sich bereits in einem der ersten Einträge im Text an, der auf den 13.03.2010 datiert ist: „Gib mir ein Jahr, Herrgott, an den ich nicht glaube, und ich werde fertig mit allem“ (Herrndorf 22). In diesem Zitat drückt sich die Verzweiflung über die Diagnose genauso aus wie die Konzentration auf die Arbeit als haltgebende Vision für die bevorstehende Zeit. Das flektierte Zahlwort alles meint dabei in erster Linie Herrndorfs Romanprojekte, denen er sich im Anschluss an seine Diagnose mit hoher Konzentration und Arbeitsintensität widmet, worin sich das Arbeiten als 
Strategie erweist, um auf den medizinischen Befund zu reagieren. Hierzu passend heißt es später im Text: „Am besten geht's mir, wenn ich arbeite“ (ebd. 44). Die Bewertung des Arbeitens als höchstes Ziel zieht sich durch die Einträge und findet unter anderem in der Sorge Ausdruck, dass die fortschreitende Krankheit für das autobiographische Ich bezwecken könnte, „nicht fertig zu werden“ (ebd. 242), was von ihm als größere Angst eingestuft wird als diejenige vor den wiederholt auftretenden neurologischen Ausfällen (ebd.). Somit fungiert die Arbeit in gewisser Weise als eine Form von Beschäftigungstherapie und bewirkt zugleich eine Verschiebung der Angst vor dem bevorstehenden Tod auf die vor verlorene Arbeitszeit (ebd. 392).

Die enge Verbindung der medizinischen Diagnosen und deren Auswirkung auf die Arbeit soll hier beispielhaft an einem Eintrag vom 21.9.2010 illustriert werden. In diesem heißt es angesichts eines bevorstehenden Untersuchungsergebnisses, von dem das autobiographische Ich vermutet, dass es sein „Todesurteil“ (ebd. 92) sein könne: „Für anschließend zwei Pläne: Wenn kein Tumorwachstum, setz ich mich an den Wüstenroman und hau ihn bis zum nächsten MRT zusammen. Im andern Fall: Werf ich ihn weg und verleg mich aufs Blog. " ${ }^{\text {" }}$ (ebd. 93). Dabei wirken sich nicht nur die medizinischen Untersuchungsergebnisse, sondern auch selbstgestellte Diagnosen, die auf Google-Recherchen basieren, auf das Befinden und somit das Arbeiten des autobiographischen Ich aus (beispielhaft ebd. 326).

In dem Zeitraum, den der autobiographische Text umfasst, unterzieht sich das autobiographische Ich wiederholt Strahlentherapien, medikamentösen Behandlungen und insgesamt drei OPs. Die MRT-Ergebnisse, die in dieser Zeit wiederholt präsentiert werden, bezwecken die unterschiedlichsten Reaktionen beim autobiographischen Ich, wobei diese immer von der hieraus resultierenden verbleibenden Zeit geprägt sind. So lassen sich als Reaktionen im Text Glücksempfinden bei einem hoffnungsversprechenden Arztgespräch (ebd. 24), ein Nervenzusammenbruch angesichts eines eingestellten Tumorwachstums (ebd. 66), Gefühle von Beunruhigung nach Überleben „de[s] größere[n] Teil[s] der statistisch erwartbaren Zeit" (ebd. 178) oder auch ein Gefühl der Erleichterung, bedingt durch die Klarheit, die das MRT-Ergebnis schafft (ebd. 246), finden. Hier verdeutlicht sich die Funktion der Zeit als von den medizinischen Untersuchungsergebnissen abhängige Entscheidensressource, die über die bei- 
den bestehenden Umgangsformen mit dem bevorstehenden Tod herrscht: die Arbeit und den Suizid.

Die Beendigung der geplanten Romanprojekte und somit das Arbeiten haben für das autobiographische Ich dabei höchste Priorität, während dem Suizid die bereits erwähnte Funktion der Exitstrategie zukommt, die dem autobiographischen Ich ein selbstbestimmtes Sterben ermöglicht (ebd. 142). Der Fokus des autobiographischen Ich liegt also eindeutig auf dem Leben und der Erfüllung der Pflicht des autobiographischen Ich, die es sich selbst in Form des Schreibens auferlegt hat (beispielhaft ebd. 161). Das zunächst formulierte Ziel, „noch ein Buch [zu] schreiben, [..]“ (ebd. 107) ändert sich mit der Bekanntmachung weiterer Diagnosen, die mehr verbleibende Zeit versprechen, sodass das autobiographische Ich das Ziel für sich im Verlauf des Textes mit der Beendigung zweier Projekte auslegt (ebd. 289) und schließlich sogar noch an einem dritten zu arbeiten beginnt (ebd. 316). Ein Leben ohne Arbeit scheint kaum denkbar. So heißt es zwar in einem mit dem 25.11.2011 datierten Eintrag: „Ich habe fast zwanzig Monate durchgearbeitet, weil ich musste. Jetzt muss ich nicht mehr. Also schreibe ich nicht mehr. Schon praktisch seit dem vierten November nicht mehr“ (ebd. 287). Nicht einmal zwei Monate später ist die Arbeit jedoch wiederaufgenommen (ebd. 294). Konkret formuliert findet sich die Beschäftigungsstrategie, die der Arbeit zukommt, in einem Eintrag vom 04.11.2011, wenn das autobiographische Ich seinen dritten Roman aus dem Grund weiterschreiben möchte, dass es ja irgendwas machen müsse: „Ich kann hier nicht rumsitzen. Noch zwölf rezidivfreie Wochen, und das Ding könnte fertig sein“ (ebd. 275).

Auch die Abhängigkeit der Suizid-Entscheidung von den medizinischen Diagnosen lässt sich anhand einer Textstelle illustrieren. So lautet ein Eintrag vom 30.04.2010: „Googeln fällt mir unsagbar schwer, ein praktikables How-to ist nicht auffindbar. Freunde informiert: Falls jemand von Mitteln und Wegen weiß oder im Besitz davon ist - am 21. Juni ist das erste MRT. Bis dahin brauche ich was hier" (ebd. 50). Dieser konkreten Formulierung gehen unterschiedliche Andeutungen voraus, die die Suizidabsicht des autobiographischen Ich erahnen lassen.

So notiert das autobiographische Ich bereits in der Rückblende Teil 4: Das Moleskine neben Kontaktdaten, Terminen und sonstigen Listen das Wort „Waffenschein" (ebd. 115) in sein Notizbuch und deutet somit vor Formulierung der Entscheidung, sich eine Waffe zu besorgen, bereits an, dass es über den Besitz 
einer solchen nachdenkt. Der visierte Waffenkauf kann hier stellvertretend für die Suizidabsicht des autobiographischen Ich gelesen werden, bevor diese konkret ausformuliert wird. Im sich anschließenden Rückblende-Kapitel HaShem wird das Motiv der Waffe erneut aufgegriffen, indem das autobiographische Ich diese als Abwehrmethode gegen seine angsteinflößenden Gedanken an den Tod einsetzt. So stellt es sich vor, eine Waffe des Models Walther PPK in seinem Kopf zu installieren, welche jeden Gedanken an den Tod erschießt und somit aus seinem Kopf verbannt (ebd. 118). An dieser Stelle wird die Idee der Waffe als Möglichkeit der Angstvermeidung im Text etabliert. ${ }^{6}$ Auch im Rückblendeteil Tanz der seligen Geister findet die Walther PPK Erwähnung, wenn das autobiographische Ich sich angesichts der von seinen Freund*innen gerufenen „Sanitäter“ (ebd. 142) ${ }^{7}$ vorstellt, dass deren Anwesenheit das Indiz für die krankheitsbedingte Auflösung seines Gehirns und einer hieraus resultierenden Veränderung seiner Persönlichkeit sei, und sich dieser Situation durch Suizid entziehen möchte:

[...] und ich weiß auch, dass ich mir für diesen Fall von Anfang an ein bestimmtes Vorgehen überlegt habe: Selbstmord, solange ich noch einen Rest von Kontrolle habe über das Gemüse, das einmal meinen Namen trug. Ich sehe die Walther PPK in meiner Hand, ich sehe sie in meinem Mund. (ebd.)

Explizit heißt es im Rahmen der Schilderung eines Traumes, in welchem das autobiographische Ich Valium zur Beruhigung schluckt und anschließend versucht, sich zu übergeben, aus Angst, die Tabletten könnten ihn „in dieser Dosierung bereits einschläfern“, „Ich trinke eine Schüssel Salzwasser und weiß: Ich brauche eine Waffe“ (ebd. 31). Das Erschießen wird somit frühzeitig als Suizidmethode im Text thematisiert, auch wenn die Entscheidung, auf welche Art und Weise der beabsichtigte Suizid letztlich erfolgen soll, noch nicht endgültig gefallen ist. Dies wird bei der Lektüre des Eintrags vom 30.04.2010 deutlich, aus dem hervorgeht, dass das autobiographische Ich seine vorherige Überzeugung, „dass es nur eine Waffe sein könne“ (ebd. 50) infrage stellt. Die finale Festlegung

\footnotetext{
${ }^{6}$ Durch die Versetzung der Rückblende-Teile trotz Erzählung einer früheren Zeit an eine spätere Stelle im Gesamttext handelt es sich innerhalb der erzählten Geschichte um die erste Erwähnung der Waffe, die somit auf den 3. oder 4. März 2010 fällt.

${ }^{7}$ Die Sanitäter werden angesichts eines „Anfalls“ des autobiographischen Ich gerufen, bei dem es rumschreit und sein Notizbuch durch den Raum wirft (Herrndorf 142).
} 
hinsichtlich der Suizidmethode lässt sich mit dem schließlich vorgenommenen Kauf der Waffe auf den 10.8.2010 datieren (ebd. 79).

Die Entscheidung, sich das Leben zu nehmen, hinterfragt das autobiographische Ich im Gegensatz zu derjenigen nach der Suizidmethode nicht, sondern erklärt die Notwendigkeit besagter Exitstrategie mit dem Umstand, dass es nicht wirklich sterben wolle, aber, wenn es dies doch müsse, dann zumindest selbst darüber zu verfügen wünsche: „Weil, ich wollte ja nicht sterben, zu keinem Zeitpunkt, und will es auch jetzt nicht. Aber die Gewissheit, es selbst in der Hand zu haben, war von Anfang an notwendiger Bestandteil meiner Psychohygiene“ (ebd. 50).

Die Möglichkeit, den eigenen Todeszeitpunkt selbst zu bestimmen, stellt für das autobiographische Ich angesichts der sonstigen Unvermeidbarkeit des krankheitsbedingten Todes einen beruhigenden Gedanken dar, mit welchem es seine Furcht davor, am Glioblastom zu sterben, bewältigen kann. Dies wird anhand eines späteren Eintrags besonders deutlich, wenn es heißt: „Ich habe mich damit abgefunden, dass ich mich erschieße. Ich könnte mich nicht damit abfinden, vom Tumor zerlegt zu werden, aber ich kann mich damit abfinden, mich zu erschießen. Das ist der ganze Trick. Schon seit Tagen keine Beunruhigung mehr." (ebd. 86)

Das Denken an die Waffe und das Arbeiten an den Romanprojekten teilen sich folglich den Platz der Bewältigungsstrategie der Angst. Entsprechend heißt es im auf den 13.5.2010 datierten Eintrag, der auf die Information folgt, dass das autobiographische Ich ein Beruhigungsmittel eingenommen hat: „Die Beruhigung setzt so schnell ein, dass man davon ausgehen muss, dass es nicht die Tablette ist, sondern die sofort nach Tabletteneinwurf konzentriert wieder aufgenommene Arbeit. Merken“ (ebd. 56). Eine ähnliche Funktion kommt der Waffe als Versinnbildlichung des geplanten Suizids zu. So löst im weiteren Verlauf des Textes das alleinige Berühren von ihr ein sicheres bzw. beruhigendes Gefühl beim autobiographischen Ich aus, welches es ihm allein ermöglicht, zu arbeiten (ebd. 247, 372) und somit seiner höchsten Priorität nachzugehen.

\section{Die Akzeptanz des Krankensuizids}

In dem bereits erwähnten Eintrag vom 30.4.2010 findet sich neben der erstmaligen Formulierung des Wunsches nach einer Exitstrategie außerdem die 
Aussage, dass das autobiographische Ich seine Freund ${ }^{\star}$ innen über seinen Suizidwunsch informiert habe (ebd. 50).

Dass es neben der Einweihung der Freund ${ }^{*}$ innen außerdem eine direkte Integration derselben in die Suizidpläne des autobiographischen Ich gibt, wird durch den Vermerk in einem späteren Eintrag deutlich, dass es die Patronen für die Waffe seiner Freundin X gibt, damit diese sie aufbewahren kann. X. nimmt somit die Position einer zu konsultierenden Instanz ein, um den Suizid final durchführen zu können (ebd. 80). Angesichts dieser direkten Integration des sozialen Umfeldes in das Vorhaben des autobiographischen Ich, ließe sich Kritik an der Suizidabsicht erwarten, die allerdings ausbleibt.

Die fehlende Schilderung von kritischen Meinungen bezüglich des Vorhabens des autobiographischen Ich durch die Freund ${ }^{*}$ innen kann dabei auf verschiedene Aspekte zurückgeführt werden und lässt sich beispielsweise mit der Textform begründen, die ein Aussparen von Kritik durch Freund ${ }^{*}$ innen nahelegt, da der Blog in seiner Ursprungsform als Informationstext für selbige gedacht war.

Eine weitere Erklärung kann in dem Verständnis des vom autobiographischen Ich visierten Suizids als Selbsttötung, die, wie eingangs geschildert, unter den Oberbegriff der „Notsuizide“ (Macho 80) gefasst werden kann, gelesen werden. Auf das Angebot einer Freundin des autobiographischen Ich, dass sie „den Rest mit der Plastiktüte erledigen [werde]“ (Herrndorf 63), sollte der Suizid durch Erschießen nicht gelingen, reagiert das autobiographische Ich als einzige Person im Raum mit gemischten Gefühlen. So heißt es am Ende des entsprechenden Eintrags „Es ist rührend, und ein bisschen graust es mich auch. Aber da bin ich anscheinend der Einzige im Raum“ (ebd.), wodurch suggeriert wird, dass seine anwesenden Freund*innen den visierten Suizid wie das autobiographische Ich selbst als unausweichliche Notwendigkeit ansehen und hinnehmen. Die Reaktionen der Freundinnen bestätigen somit die eingangs als erwartbar beschriebene Legitimation der krankheitsbedingten Suizidabsicht. So lassen sie nie auf die implizierte Absicht schließen, das autobiographische Ich von seinem visierten Suizid abhalten zu wollen, sondern drücken lediglich ihre Emotionen angesichts des bevorstehenden Todes aus. Ob dieser selbst herbeigeführt wird oder aufgrund der bestehenden Krankheit erfolgt, scheint keine Rolle zu spielen. Allerdings ist auch hier erneut auf den Umstand zu verweisen, dass die Möglichkeit von Aussparungen innerhalb des Textes besteht. 


\section{Schlusswort}

Wie eingangs erwähnt, fungiert die Suizidabsicht des autobiographischen Ich neben dem Arbeiten als Bewältigungsstrategie für das diagnostizierte Glioblastom. Sie sichert dem autobiographischen Ich Handlungsmacht in einer Zeit, in der der Verlust von Kontrolle die größte Angst darstellt und ermöglicht angesichts der tödlichen Erkrankung einen letzten Akt der Selbstbestimmung. Die Entscheidung zur Selbsttötung ist dabei bereits zu Beginn des Textes gefallen und bleibt lediglich hinsichtlich der Frage nach dem richtigen Zeitpunkt für den Vollzug prozesshaft. Die herangezogenen Statistiken, eigen vorgenommenen Recherchen, Ärzt*innen-Gespräche und immer neuen medizinischen Untersuchungsergebnisse stellen diesbezüglich relevante Entscheidensressourcen dar, die immer mit der mit ihnen einhergehenden Lebenserwartung korrelieren. Das Arbeiten bleibt primäres Ziel, der Suizid ist letztendlich nur die unvermeidliche Reaktion für „ein[en] letzte[n] Triumph des Geistes über das Gemüse“ (ebd. 198).

Arbeit und Struktur endet mit einem letzten Eintrag am 20.8.2013 (ebd. 425). Im Nachwort, das sich in der Taschenbuchausgabe befindet, heißt es: „Wolfgang Herrndorf hat es gemacht, wie es zu machen ist. Am Montag, den 26. August [2013, Anm. ZR] gegen 23:15 schoss er sich am Ufer des Hohenzollernkanals mit einem Revolver in den Kopf. [...] Es dürfte einer der letzten Tage gewesen sein, an denen er noch zu der Tat imstande war" (Gärtner und Passig 445).

Der „point of no return“ (Lübbe 21) ist erreicht: Das Ende des Autors und somit des autobiographischen Ich bedingt das Ende des Textes. Die letzten Sätze dieses Papers sollen ebenfalls dem autobiographischen Ich gehören. Sie stammen aus dem Eintrag vom 28.3.2013, in dem eine erneute Untersuchung das Wachstum eines „spiegelbildlich zum Glioblastom gelegene[n] Tumor[s]“ (Herrndorf 402) zeigt und eröffnen die Perspektive, dass Arbeit und Struktur nicht nur ein Tagebuchblog, sondern letztlich auch eine eigene Statistik ist: „Eine Prognose gibt es nicht, eine allgemeine Statistik auch nicht. Nach drei OPs, zwei Bestrahlungen, drei verschiedenen Chemos ist man seine eigene Statistik“ (ebd.). 


\section{Literaturverzeichnis}

\section{Primärliteratur}

Herrndorf, Wolfgang. Arbeit und Struktur. Rowohlt, 2013.

---. Arbeit und Struktur. www.wolfgang-herrndorf.de/ (zuletzt aufgerufen am 22.11.2021).

\section{Sekundärliteratur}

Améry, Jean. Hand an sich legen. Diskurs über den Freitod. 7. Aufl. Klett-Cotta, 1983.

Aristoteles. Die Nikomachische Ethik. Hrsg. von Rainer Nickel. 2. Aufl. Artemis \& Winkler, 2007.

Gärtner, Marcus und Kathrin Passig. „Nachwort“. Arbeit und Struktur. Wolfgang Herrndorf. Rowohlt, 2013, S. 443-445.

Graefe, Annette. Das Suizidmotiv in der deutschsprachigen Literatur. Gestaltung und Funktion. Dup, 2017.

Kant, Immanuel. Grundlegung zur Metaphysik der Sitten. 4. Aufl. Hartknoch, 1797.

Lübbe, Hermann. „Zur Theorie der Entscheidung“, in: Theorie und Entscheidung. Studien zum Primat der praktischen Vernunft. Rombach, 1971, S. 7-31.

Macho, Thomas. Das Leben nehmen. Suizid in der Moderne. Suhrkamp, 2017.

Minois, Georges. Geschichte des Selbstmords. Artemis \& Winkler, 1996.

Pfister, Ulrich. „Einleitung“, in: ders. (Hrsg.). Kulturen des Entscheidens. Narrative - Praktiken - Ressourcen. Vandenhoeck \& Ruprecht, 2019, S. 11-34.

Platon. „Phaidon“. Sämtliche Werke, 1. Lambert Schneider 1950, S. 731-811.

Decher, Friedhelm. Die Signatur der Freiheit. Ethik des Selbstmords in der abendländischen Philosophie. Zu Klampen, 1999.

Seneca, Lucius Annaeus. Briefe an Lucilius. Marion Giebel (Hg.). Reclam, 2014.

Vorjans, Gerrit. Von der Torheit, wählerisch zu sterben. Suizid in der deutschsprachigen Literatur um 1900. Transcript, 2016.

Wagner-Egelhaaf, Martina. Autobiographie. 2. aktualisierte und erweiterte Aufl. J.B. Metzler, 2005.

---. „Trauerspiel und Autobiographie. Handeln und Entscheiden bei Goethe“. Kulturen des Entscheidens. Narrative - Praktiken - Ressourcen. Ulrich Pfister (Hg). Vandenhoeck \& Ruprecht, 2019, S. 71-89. 


\title{
BEATING DEATH BY DYING. SUICIDE AS AN EXIT STRATEGY IN WOLFGANG HERRNDORF'S ARBEIT UND STRUKTUR
}

\begin{abstract}
Zarah RIETSCHEL

Westfälische-Wilhelms-Universität Münster, Germany

Schlosspl. 2, 48149 Münster

zarahrietschel@gmail.com
\end{abstract}

This paper provides a textual analysis of Wolfgang Herrndorf's Arbeit und Struktur regarding the connection between the autobiographical self's decision to commit suicide and his diagnosis of a fatal brain tumor (glioblastoma). It shows that the repeated examinations and their results, as well as consulted statistics and self-conducted research, function as the so-called "decision resources" regarding the intention to commit suicide. They affect thereby the endurance as well as the question concerning an eligible date for the intended suicide. In addition to studying different reactions to the course of the disease using the method of close reading, this paper undertakes a parallelization of the expressed suicidal intention and the autobiographical self's intensive work as forms of a coping strategy. Working, more precisely writing, acts primarily as a meaningful occupation, while the planned suicide functions as an "exit strategy" that is used as the last possible solution to fight the illness. While a variety of interdisciplinary discourses have always rejected 'suicide' as a self-determined decision, suicide due to illness experiences a historically persistent legitimation. This can also be observed in the studied text on the reactions of the autobiographical self's social environment. The inevitable death in the face of medical diagnoses thus legitimizes suicide.

Keywords: Wolfgang Herrndorf, Arbeit und Struktur, autobiography, suicide, disease 


\title{
POBIJEDITI SMRT SMRĆU. SAMOUBOJSTVO KAO IZLAZ U ARBEIT UND STRUKTUR WOLFGANGA HERRNDORFA
}

\begin{abstract}
Sažetak
Zarah RIETSCHEL

Westfälische-Wilhelms-Universität Münster

Schlosspl. 2, 48149 Münster

zarahrietschel@gmail.com
\end{abstract}

Rad donosi analizu knjige Arbeit und Struktur Wolfganga Herrndorfa o vezi između odluke o samoubojstvu autobiografskoga ja i njegove dijagnoze neizlječivog tumora mozga (glioblastom). Pokazuje se kako neprestane pretrage i njihovi rezultati, kao i proučena statistika i samostalno istraživanje, funkcioniraju kao tzv. „resursi odluke“ pri namjeri samoubojstva, pri čemu utječu na otpornost te na odabir prikladnog nadnevka za namjeravano samoubojstvo. Osim analize različitih reakcija na tijek bolesti zahvaljujući metodi pomnog čitanja, rad iznalazi paralele između izražene samoubilačke namjere i intenzivnog rada autobiografskoga ja kao strategije suočavanja. Predanost radu, točnije pisanju, djeluje ponajprije kao smisleno zanimanje, dok planirano samoubojstvo funkcionira kao „izlazna strategija“ koja se rabi kao krajnje rješenje u borbi protiv bolesti. I dok su različiti interdisciplinarni diskursi „samoubojstvo“ oduvijek odbacivali kao osobnu odluku, samoubojstvo zbog bolesti tijekom povijesti je dosljedno obilježeno opravdanošću, što je u tekstu također moguće uočiti po reakcijama društvenog okruženja autobiografskoga ja. Neizbježna smrt u jeku medicinskih dijagnoza na taj način opravdava samoubojstvo.

Ključne riječi: Wolfgang Herrndorf, Arbeit und Struktur, autobiografija, samoubojstvo, bolest 